\title{
Adaptive-Impulsive Control of the Projective Synchronization in Drive-Response Complex Dynamical Networks with Time-Varying Coupling
}

\author{
Song Zheng ${ }^{1,2}$ \\ ${ }^{1}$ School of Mathematics and Statistics, Zhejiang University of Finance and Economics, Hangzhou, \\ Zhejiang 310018, China \\ ${ }^{2}$ Faculty of Science, Jiangsu University, Zhenjiang, Jiangsu 212013, China
}

Correspondence should be addressed to Song Zheng, songzheng07318@yahoo.com.cn

Received 25 February 2011; Accepted 6 July 2011

Academic Editor: Zidong Wang

Copyright (C) 2012 Song Zheng. This is an open access article distributed under the Creative Commons Attribution License, which permits unrestricted use, distribution, and reproduction in any medium, provided the original work is properly cited.

This paper investigates the projective synchronization (PS) of drive-response time-varying coupling complex dynamical networks with time delay via an adaptive-impulsive controlling method, in which the weights of links are time varying. Based on the stability analysis of impulsive control system, sufficient conditions for the PS are derived, and a hybrid controller, that is, an adaptive feedback controller with impulsive control effects, is designed. Numerical simulations are performed to verify the correctness and effectiveness of theoretical result.

\section{Introduction}

Complex network models widely exist in the real world including the spread of infectious diseases, the World Wide Web, food webs, various wireless communication networks, metabolic networks, biological neural works, and scientific citation webs. Since the discovery of small-world effect [1] and scale-free feature [2] of complex networks, many scientists and engineers from various disciplines, such as mathematics, physics, biology, engineering, have paid increasing attention to the studies of complex networks.

Synchronization, one of the typical collective behaviours of complex dynamical networks, has received rapidly increasing attention from different fields in recent years [324]. And different control schemes including adaptive control [10-12], pinning control [1316], and impulsive control [17-22] have been used to study the above problem. Recently, some authors presented hybrid control strategy to investigate the synchronization of complex networks [23-26]. Compared to the conventional control method, hybrid control method is more effective to the networks with evolutionary features [23]. In many practical situations, some complex networks may change suddenly and sharply and thus the modes switch 
simultaneously. This kind of networks can be found in many evolutionary processes, such as optimal control model in economics, bursting rhythm model in pathology, mobile communication networks, and social networks.

The typical configuration of chaotic synchronization consists of drive and response systems and has been widely investigated. The PS, a new chaos synchronization phenomenon, was first studied in two coupled partially linear systems by Mainieri and Rehacek in [27]. Later, the PS between two complex networks has obtained much more attention [2832]. In [28], Guo et al. studied the PS in drive-response networks via impulsive control. In $[29,30]$, authors discussed the PS of a drive-response dynamical network model without the time delay. Sun et al. studied the PS in drive-response dynamical networks of partially linear systems with time-varying coupling delay in [31]. Xu et al. [32] investigated the PS of a class of the drive-response dynamical networks with coupling delays. However, the time-varying coupling was not considered in the above studies. To simulate more realistic networks, timevarying coupling [33-35] should be taken into account. Motivated by the above discussions, this paper will focus on the adaptive-impulsive PS problem of drive-response time-varying coupling dynamical networks with time delay. Based on the stability theory of the impulsive differential equation, some criteria for the PS are derived. Furthermore, analytical results show that the drive-response networks can realize the PS.

The rest of this paper is organized as follows. In Section 2, we present the model of drive-response time-varying coupling dynamical networks with time delay and a hybrid controller is designed. In Section 3, synchronization criteria for PS are derived. Numerical simulations are shown in Section 4. The conclusion is finally drawn in Section 5.

\section{Model Description and Preliminaries}

Consider the following drive-response time-varying coupling dynamical network model with time delay:

$$
\begin{gathered}
\dot{u}_{d}(t)=M(z) \cdot u_{d}(t), \\
\dot{z}(t)=f\left(u_{d}(t), z(t)\right), \\
\dot{u}_{r i}(t)=M(z) \cdot u_{r i}(t)+c \sum_{j=1}^{N} c_{i j}(t) \Gamma(t) u_{r j}(t-\tau), \quad i=1,2, \ldots, N,
\end{gathered}
$$

where the drive system and the response network systems are linked through the variable $z(t) \in R^{1}, u_{d}(t)=\left(u_{d}^{1}(t), u_{d}^{2}(t), \ldots, u_{d}^{n}(t)\right)^{T} \in R^{n}, u_{r i}(t)=\left(u_{r i}^{1}(t), u_{r i}^{2}(t), \ldots, u_{r i}^{n}(t)\right)^{T} \in R^{n}$ and the $d$ and $r$ stand for the drive system and response system, respectively. The constant $c>0$ is the coupling strength to be adjusted, $\tau \geq 0$ is the time-delay. $M(z) \in R^{n \times n}$ is a matrix which depends on the variable $z(t) . \Gamma(t) \in R^{n \times n}$ is the time-varying inner-coupling link matrix at time $t$. $C(t)=\left(c_{i j}(t)\right)_{N \times N}$ is the outer-coupling configuration matrix, in which $c_{i j}(t) \neq 0$ if there is a link from node $i$ to node $j(i \neq j)$, and $c_{i j}(t)=0(i \neq j)$ otherwise, the diagonal elements of matrix $C(t)$ are given by

$$
c_{i i}(t)=-\sum_{j=1, j \neq i}^{N} c_{i j}(t), \quad i=1,2, \ldots, N .
$$

If there exists a constant $\alpha(\alpha \neq 0)$ such that $\lim _{t \rightarrow \infty}=\left\|e_{i}(t)\right\|=\left\|u_{r i}(t)-\alpha u_{d}(t)\right\|=0$ for $i=1,2, \ldots, N$, then the PS of network (2.1) is achieved. $\alpha$ is a desired scaling factor. 
The aim of this paper is to discuss the adaptive-impulsive PS in the drive-response timevarying coupling complex dynamical network with time delay. We choose the linear impulsive controller $B_{i_{k}}$ which is a $n \times n$ constant matrix, and design an adaptive feedback controller $U_{i}$. Therefore, the network (2.1) can be rewritten as the following impulsive differential equations:

$$
\begin{gathered}
\dot{u}_{d}(t)=M(z) \cdot u_{d}(t), \\
\dot{z}(t)=f\left(u_{d}(t), z(t)\right), \\
\dot{u}_{r i}(t)=M(z) \cdot u_{r i}(t)+c \sum_{j=1}^{N} c_{i j}(t) \Gamma(t) u_{r j}(t-\tau)+U_{i}, \quad t \neq t_{k}, \\
\Delta u_{r i}=u_{r i}\left(t_{k}^{+}\right)-u_{r i}\left(t_{k}^{-}\right)=B_{i_{k}}\left[u_{r i}-\alpha u_{d}\right], \quad t=t_{k},
\end{gathered}
$$

where $u_{r i}\left(t_{k}^{+}\right)=\lim _{t \rightarrow t_{k}^{+}} u_{r i}(t), u_{r i}\left(t_{k}^{-}\right)=\lim _{t \rightarrow t_{k}^{-}} u_{r i}(t)$. Moreover, any solution of (2.3) is left continuous at each $t_{k}$, that is, $u_{r i}\left(t_{k}^{-}\right)=u_{r i}\left(t_{k}\right)$.

Letting the PS error $e_{i}(t)=u_{r i}(t)-\alpha u_{d}(t)$, the adaptive controllers $U_{i}$ and updating laws are designed as follows:

$$
\begin{gathered}
U_{i}=-d_{i} e_{i}(t), \\
\dot{d}_{i}=k_{i} e_{i}^{T}(t) e_{i}(t)=k_{i}\left\|e_{i}(t)\right\|^{2}, \quad k_{i}>0 .
\end{gathered}
$$

The system (2.3) is said to be synchronized if $\lim _{t \rightarrow \infty}\left\|e_{i}(t)\right\|=0$. by

Under the adaptive-impulsive control, the error dynamical network is characterized

$$
\begin{gathered}
\dot{e}_{i}(t)=M(z) \cdot e_{i}(t)+c \sum_{j=1}^{N} c_{i j}(t) \Gamma(t) e_{j}(t-\tau)+U_{i}, \quad t \neq t_{k} \\
\dot{z}(t)=f\left(u_{d}(t), z(t)\right) \\
\Delta e_{i}=B_{i_{k}} e_{i}, \quad t=t_{k}, \quad k=1,2, \ldots
\end{gathered}
$$

Before proceeding, we give some necessary assumptions and lemmas to derive the main results of the paper.

Lemma 2.1. The matrix inequality $2 x^{T} y \leq x^{T} Q x+y^{T} Q^{-1} y$ holds, for any vectors $x, y \in R^{n}$ and $a$ positive-definite matrix $Q \in R^{n \times n}$.

Assumption 2.2. Suppose there exists a positive constant $\xi$, such that $\left\|e_{i}(t-\tau)\right\|^{2} \leq \xi\left\|e_{i}(t)\right\|^{2}$ holds.

\section{PS Analysis}

In this section, we will make drive-response time-varying coupling complex dynamical networks achieve the PS by using the adaptive-impulsive controlling method. 
Theorem 3.1. For given synchronization scaling factor $\alpha$, the PS in drive-response dynamical networks will occur if the following conditions hold:

(i) If there exist two constants $a, \lambda$ satisfying $0 \leq a<\lambda$ and $\delta(t) \leq-\lambda<0$ such that

$$
\ln \left(\beta_{k}\right)-a\left(t_{k}-t_{k-1}\right) \leq 0, \quad k=1,2, \ldots,
$$

then the trivial solution of error system (2.5) is global asymptotically stable, which implies driveresponse networks achieve the projective synchronization under the adaptive-impulsive control.

(ii) If $\delta(t) \geq 0$ and there exists a constant $a \geq 1$ such that

$$
\ln \left(a \beta_{k}\right)+\int_{t_{k}}^{t_{k+1}} \delta(s) d s \leq 0, \quad k=1,2, \ldots
$$

then $a=1$ implies that the trivial solution of error system (2.5) is stable and $a>1$ implies that the trivial solution of error system (2.5) is global asymptotically stable where

$$
\begin{gathered}
\delta(t)=\max _{1 \leq i \leq N}\left\{\left(\frac{1}{\lambda_{\min }(P)}\right) \sup \left[\lambda_{\max }\left(P M(z)+M^{T}(z) P-2 d^{*} P\right)\right]+N \xi,\right. \\
\left.+\sum_{j=1}^{N}\left(c c_{i j}(t)\right)^{2}\|P \Gamma(t)\|^{2}\right\}, \\
\max _{k}\left(\left\|I+B_{i_{k}}\right\|^{2}\right)=\rho_{k}<1, \quad \beta_{k} \geq\left(\frac{\rho_{k}\|P\|}{\lambda_{\min }(P)}\right),
\end{gathered}
$$

$P$ is a positive-definite matrix. $d^{*}$ is the minimum value of the initial feedback strength $d_{i 0}\left(d_{i 0} \leq d_{i}\right)$, $i=1,2, \ldots, N$.

Proof. Consider the following Lyapunov functional:

$$
V(t)=\frac{1}{2} \sum_{i=1}^{N} e_{i}^{T}(t) P e_{i}(t)
$$

For $t \neq t_{k}$, the derivative of $V(t)$ along the trajectories of (2.5) is

$$
\begin{aligned}
\dot{V}(t)= & \frac{1}{2} \sum_{i=1}^{N} \dot{e}_{i}^{T}(t) P e_{i}(t)+\frac{1}{2} \sum_{i=1}^{N} e_{i}^{T}(t) P \dot{e}_{i}(t) \\
= & \frac{1}{2} \sum_{i=1}^{N}\left[M(z) e_{i}(t)+c \sum_{j=1}^{N} c_{i j}(t) \Gamma(t) e_{j}(t-\tau)-d_{i} e_{i}(t)\right]^{T} P e_{i}(t) \\
& +\frac{1}{2} \sum_{i=1}^{N} e_{i}^{T}(t) P\left[M(z) e_{i}(t)+c \sum_{j=1}^{N} c_{i j}(t) \Gamma(t) e_{j}(t-\tau)-d_{i} e_{i}(t)\right]
\end{aligned}
$$


Mathematical Problems in Engineering

$$
\begin{aligned}
= & \frac{1}{2} \sum_{i=1}^{N} e_{i}^{T}(t)\left[P M(z)+M^{T}(z) P-2 d_{i} P\right] e_{i}(t)+\frac{1}{2} \sum_{i=1}^{N} \sum_{j=1}^{N} e_{j}^{T}(t-\tau) c c_{i j}(t) \Gamma^{T}(t) P e_{i}(t) \\
& +\frac{1}{2} \sum_{i=1}^{N} \sum_{j=1}^{N} e_{i}^{T}(t) c c_{i j}(t) P \Gamma(t) e_{j}(t-\tau) \\
\leq & \frac{1}{2} \sum_{i=1}^{N}\left\{\sup \left[\lambda_{\max }\left(P M(z)+M^{T}(z) P-2 d_{i} P\right)\right]\right\} e_{i}^{T}(t) e_{i}(t) \\
& +\frac{1}{2} \sum_{i=1}^{N} \sum_{j=1}^{N} e_{i}^{T}(t)\left(c c_{i j}(t)\right)^{2} P \Gamma(t) \Gamma^{T}(t) P^{T} e_{i}(t) \\
& +\frac{1}{2} \sum_{i=1}^{N} \sum_{j=1}^{N} e_{j}^{T}(t-\tau) e_{j}(t-\tau) \\
\leq & \frac{1}{2} \sum_{i=1}^{N}\left\{\sup \left[\lambda_{\max }\left(P M(z)+M^{T}(z) P-2 d_{i 0} P\right)\right]\right\} e_{i}^{T}(t) e_{i}(t) \\
& +\frac{1}{2} \sum_{i=1}^{N} \sum_{j=1}^{N} e_{i}^{T}(t)\left(c c_{i j}(t)\right)^{2} P \Gamma(t) \Gamma^{T}(t) P^{T} e_{i}(t)+\frac{N}{2} \sum_{i=1}^{N} e_{i}^{T}(t-\tau) e_{i}(t-\tau) .
\end{aligned}
$$

From Assumption 2.2, we get

$$
\frac{N}{2} \sum_{i=1}^{N} e_{i}^{T}(t-\tau) e_{i}(t-\tau) \leq \frac{N \xi}{2} \sum_{i=1}^{N} e_{i}^{T}(t) e_{i}(t) .
$$

Thus we have

$$
\begin{aligned}
& \dot{V}(t) \leq \frac{1}{2} \sum_{i=1}^{N}\left\{\sup \left[\lambda_{\max }\left(P M(z)+M^{T}(z) P-2 d^{*} P\right)\right]+N \xi\right. \\
&\left.+\sum_{j=1}^{N}\left(c c_{i j}(t)\right)^{2}\|P \Gamma(t)\|^{2}\right\} e_{i}^{T}(t) e_{i}(t) \\
& \leq \max _{1 \leq i \leq N}\left\{\frac{1}{\lambda_{\min }(P)} \sup \left[\lambda_{\max }\left(P M(z)+M^{T}(z) P-2 d^{*} P\right)\right]+N \xi\right. \\
&\left.\quad+\sum_{j=1}^{N}\left(c c_{i j}(t)\right)^{2}\|P \Gamma(t)\|^{2}\right\} \sum_{i=1}^{N} \frac{1}{2} e_{i}^{T}(t) P e_{i}(t) \\
&=\delta(t) \sum_{i=1}^{N} \frac{1}{2} e_{i}^{T}(t) P e_{i}(t) \\
&=\delta(t) V(t) .
\end{aligned}
$$


This implies that

$$
V(t) \leq\left(V t_{k-1}^{+}\right) \exp \int_{t_{k-1}}^{t} \delta(s) d s, \quad k=1,2, \ldots, t \in\left(t_{k-1}, t_{k}\right]
$$

When $t=t_{k}$, we have

$$
\begin{aligned}
V\left(t_{k}^{+}\right) & =\frac{1}{2} \sum_{i=1}^{N} e_{i}^{T}(t)\left(I_{2}+B_{i_{k}}\right)^{T} P\left(I_{2}+B_{i_{k}}\right) e_{i}(t) \\
& \leq \frac{\rho_{k}\|P\|}{\lambda_{\min }(P)} V\left(t_{k}\right) \\
& \leq \beta_{k} V\left(t_{k}\right), \quad k=1,2, \ldots
\end{aligned}
$$

When $k=1$ in inequality (3.8), then for any $t \in\left(t_{0}, t_{1}\right]$,

$$
V(t) \leq V\left(t_{0}^{+}\right) \exp \int_{t_{0}}^{t} \delta(s) d s
$$

This leads to

$$
V\left(t_{1}\right) \leq V\left(t_{0}^{+}\right) \exp \int_{t_{0}}^{t_{1}} \delta(s) d s
$$

Also from (3.9) we have

$$
V\left(t_{1}^{+}\right) \leq \beta_{1} V\left(t_{1}\right) \leq \beta_{1} V\left(t_{0}^{+}\right) \exp \int_{t_{0}}^{t_{1}} \delta(s) d s
$$

In the same way for $t \in\left(t_{1}, t_{2}\right]$, we have

$$
\begin{aligned}
V(t) & \leq V\left(t_{1}^{+}\right) \exp \int_{t_{1}}^{t} \delta(s) d s \\
& \leq \beta_{1} V\left(t_{0}^{+}\right) \exp \int_{t_{0}}^{t_{1}} \delta(s) d s \exp \int_{t_{1}}^{t} \delta(s) d s, \\
& =\beta_{1} V\left(t_{0}^{+}\right) \exp \int_{t_{0}}^{t} \delta(s) d s .
\end{aligned}
$$

In general for any $t \in\left(t_{k}, t_{k+1}\right]$, one finds that

$$
V(t) \leq \beta_{1} \beta_{2} \cdots \beta_{k} V\left(t^{+}\right) \exp \int_{t_{0}}^{t} \delta(s) d s
$$


(i) If there exist two constants $a, \lambda$ satisfying $0 \leq a<\lambda, \delta(t) \leq-\lambda<0$ and (3.1), we have

$$
\beta_{k} \leq e^{a\left(t_{k}-t_{k-1}\right)}, \quad k=1,2, \ldots
$$

From (3.14), one finds that

$$
\begin{aligned}
V(t) & \leq \beta_{1} \beta_{2} \cdots \beta_{k} V\left(t_{0}^{+}\right) e^{-\lambda\left(t-t_{0}\right)} \\
& =\beta_{1} \beta_{2} \cdots \beta_{k} V\left(t_{0}^{+}\right) e^{-a\left(t-t_{0}\right)-(\lambda-a)\left(t-t_{0}\right)} \\
& \leq \beta_{1} \beta_{2} \cdots \beta_{k} V\left(t_{0}^{+}\right) e^{-a\left(t_{k}-t_{0}\right)-(\lambda-a)\left(t-t_{0}\right)} \\
& \leq V\left(t_{0}^{+}\right) e^{a\left(t_{1}-t_{0}\right)} e^{a\left(t_{2}-t_{1}\right)} \cdots e^{a\left(t_{k}-t_{k-1}\right)} e^{-a\left(t_{k}-t_{0}\right)-(\lambda-a)\left(t-t_{0}\right)} \\
& =V\left(t_{0}^{+}\right) e^{-(\lambda-a)\left(t-t_{0}\right)}, \quad t \in\left(t_{k}, t_{k+1}\right] .
\end{aligned}
$$

Then the trival solution of error system (2.5) is global asymptotically stable, which implies drive-response time-varying coupling dynamical networks (2.3) achieve the PS under the adaptive-impulsive control.

(ii) If $\delta(t) \geq 0$ and there exists a constant $a \geq 1$ satisfying (3.2), we have

$$
\beta_{k} \leq \frac{1}{a} \exp \int_{t_{k}+1}^{t_{k}} \delta(s) d s
$$

From (3.14), we have

$$
\begin{aligned}
V(t) & \leq \beta_{1} \beta_{2} \cdots \beta_{k} V\left(t_{0}^{+}\right) \exp \int_{t_{0}}^{t} \delta(s) d s \\
& \leq \beta_{1} \beta_{2} \cdots \beta_{k} V\left(t_{0}^{+}\right) \exp \int_{t_{0}}^{t_{k+1}} \delta(s) d s \\
& \leq V\left(t_{0}^{+}\right) \frac{1}{a} \exp \int_{t_{2}}^{t_{1}} \delta(s) d s \frac{1}{a} \exp \int_{t_{3}}^{t_{2}} \delta(s) d s \cdots \frac{1}{a} \exp \int_{t_{k+1}}^{t_{k}} \delta(s) d s \exp \int_{t_{0}}^{t_{k+1}} \delta(s) d s \\
& =V\left(t_{0}^{+}\right) \frac{1}{a^{k}} \exp \int_{t_{0}}^{t_{1}} \delta(s) d s, \quad t \in\left(t_{k}, t_{k+1}\right] .
\end{aligned}
$$

This implies that error system (2.5) is global asymptotically stable about zero. Therefore, the PS of the drive-response dynamical networks (2.3) is achieved. The proof is completed. 
Remark 3.2. The conditions given by Theorem 3.1 do not require the network configuration to be symmetric and irreducible, which can be applied to more real-world dynamical networks. Moreover, Theorem 3.1 does not impose any bound on the time-delay constant $\tau$. Thus, our synchronization results are a time-delay-independent stability criteria.

\section{Numerical Simulation}

In this section, to verify and demonstrate the effectiveness of the proposed methods, we consider the unified chaotic system as the drive system. It is well known that the unified chaotic system is described by

$$
\begin{gathered}
\dot{x}_{1}=(25 \theta+10)\left(x_{2}-x_{1}\right), \\
\dot{x}_{2}=(28-35 \theta) x_{1}-x_{1} z+(29 \theta-1) x_{2}, \\
\dot{z}=x_{1} x_{2}-\frac{8+\theta}{3} z,
\end{gathered}
$$

where $M(z)=\left(\begin{array}{cc}-(25 \theta+10) & 25 \theta+10 \\ 28-35 \theta-z & 29 \theta-1\end{array}\right), f(x, z)=x_{1} x_{2}-(8+\theta / 3) z, x=\left(x_{1}, x_{2}\right)^{T}, \theta \in[0,1]$. System (4.1) especially is always chaotic in the whole interval $\theta \in[0,1]$.

The drive-response time-varying coupling dynamical networks with time delay are described as follows:

$$
\begin{gathered}
\dot{x}=(25 \theta+10)(y-x), \\
\dot{y}=(28-35 \theta-z) x+(29 \theta-1) y, \\
\dot{z}=x y-\frac{8+\theta}{3} z, \\
\dot{x}_{i}=(25 \theta+10)\left(y_{i}-x_{i}\right)+c \sum_{j=1}^{5} c_{i j}(t) x_{j}(t-\tau)+u_{i 1}, \\
\dot{y}_{i}=(28-35 \theta-z) x_{i}+(29 \theta-1) y_{i}+c \sum_{j=1}^{5} c_{i j}(t) x_{j}(t-\tau)+u_{i 2} .
\end{gathered}
$$

Choose the time-varying coupling configuration matrix:

$$
C(t)=\left(\begin{array}{ccccc}
-2 \sin t & -1 & 0 & 2 \sin t & 1 \\
\sin t & -\sin t-\cos t & 0 & 0 & \cos t \\
0 & \cos t & 0 & -\cos t & 0 \\
0 & \sin t \cos t & 1 & -\sin t \cos t & -1 \\
-1 & 2 \sin t & 0 & -1 & 2-2 \sin t
\end{array}\right)
$$




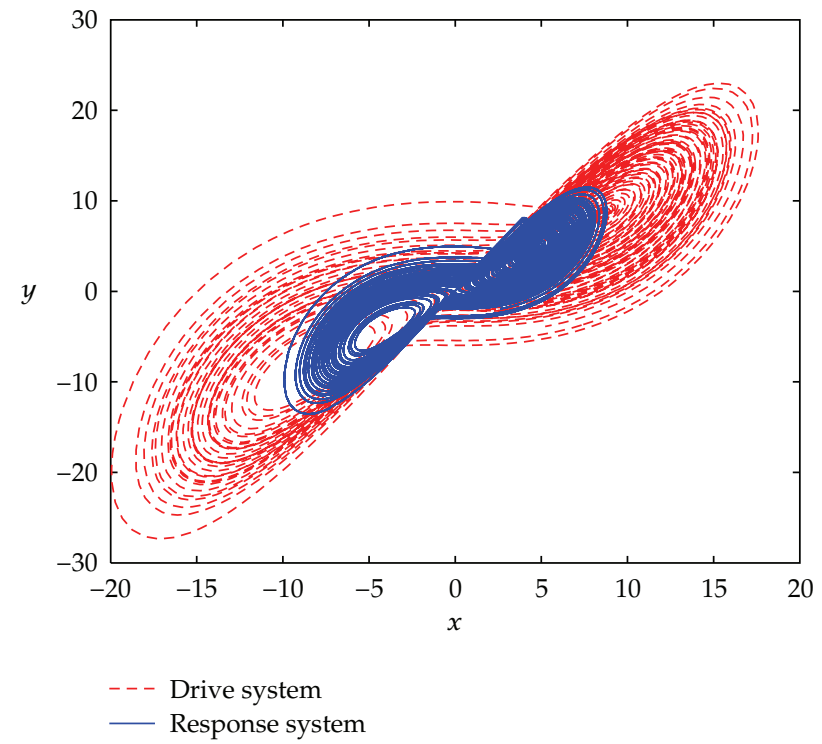

Figure 1: The trajectories of PS in the $x-y$ plane.

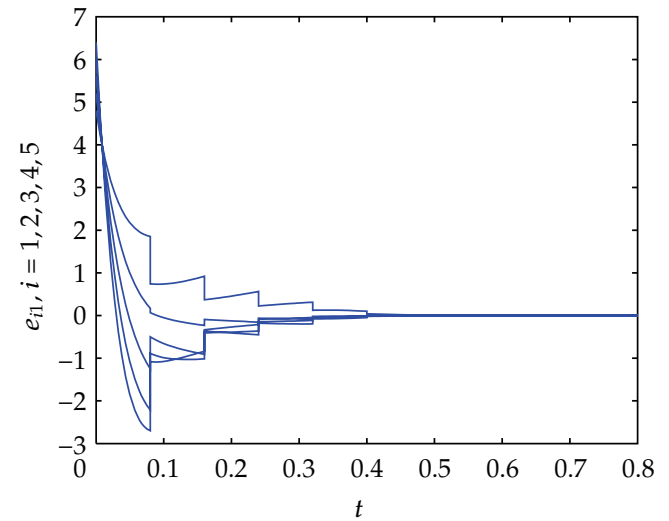

(a)

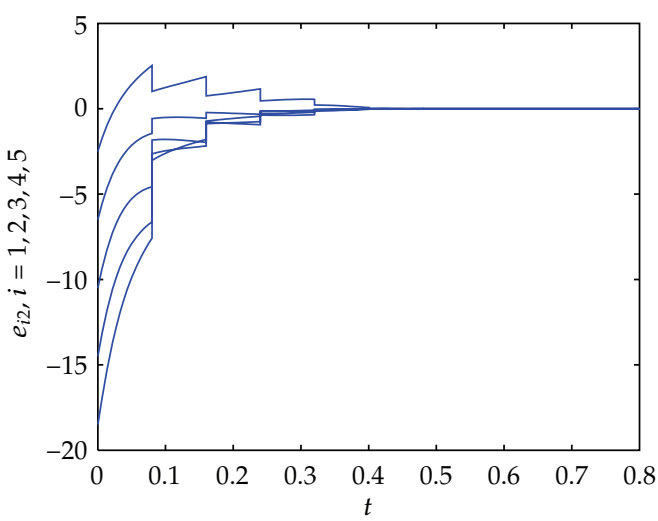

(b)

Figure 2: PS errors under the adaptive-impulsive control: (a) $e_{i 1}(t)=x_{i 1}-0.5 x_{1}$, (b) $e_{i 2}(t)=x_{i 2}-0.5 x_{2}$.

For simplicity, in the numerical simulations, we assume $P=\Gamma(t)=I_{2}, c=0.1, k_{i}=1$, $\xi=1, \theta=0.03$, the time-delay $\tau=0.07$, and the impulsive interval $t_{k+1}-t_{k}=0.08$. The initial values of the drive systems and the response systems are chosen as $-2,1,2,3.8,-2$, $4.2,-6,4.6,-10,5,-14,5.4,-18, B_{i_{k}}=\operatorname{diag}\{-0.79,-0.79\}, \beta_{k}=0.0441>0, d_{i 0}=5$. After calculations, we get $\delta(t)=\max _{1 \leq i \leq N}\left\{\left(1 / \lambda_{\min }(P)\right) \sup \left[\lambda_{\max }\left(P M(z)+M^{T}(z) P-2 d^{*} P\right)\right]+\right.$ $\left.N \xi+\sum_{j=1}^{N}\left(c c_{i j}(t)\right)^{2}\|P \Gamma(t)\|^{2}\right]=31.667>0$. Let $a=1.02$. Then $\ln \left(a \beta_{k}\right)+\int_{t_{k}}^{t_{k-1}} \delta(s) d s=$ $-0.5681<0$, according to Theorem 3.1, and the trivial solution of error system (2.5) is global asymptotically stable. Therefore, the adaptive-impulsive PS of the drive-response dynamical networks (2.3) is achieved. Figure 1 displays the trajectories of PS in the $x-y$ plane when $\alpha=$ 0.5 . The synchronization errors $e_{i 1}(t)=x_{i 1}-0.5 x_{1}$ and $e_{i 2}(t)=x_{i 2}-0.5 x_{2}(i=1,2, \ldots, 5)$ are shown, respectively, in Figure 2. Figure 3 show the evolution of the feedback strength $d_{i}$. 


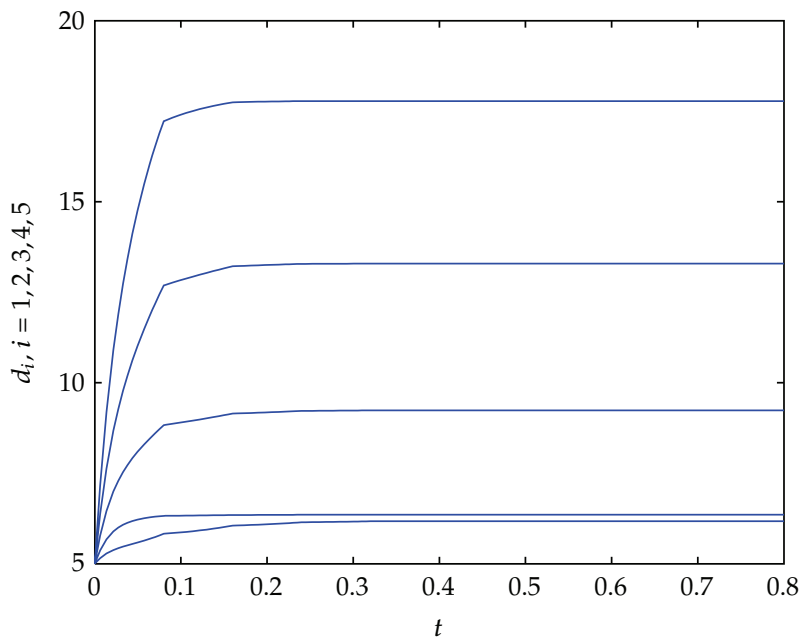

Figure 3: The evolution of the feedback strength $d_{i}, i=1,2, \ldots, 5$.

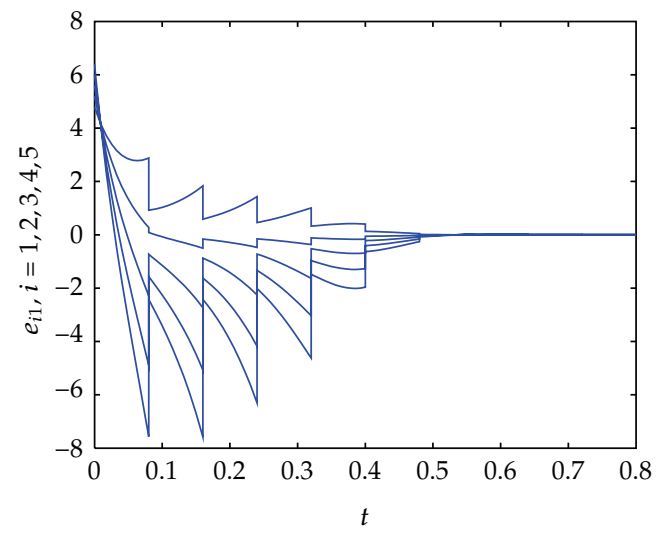

(a)

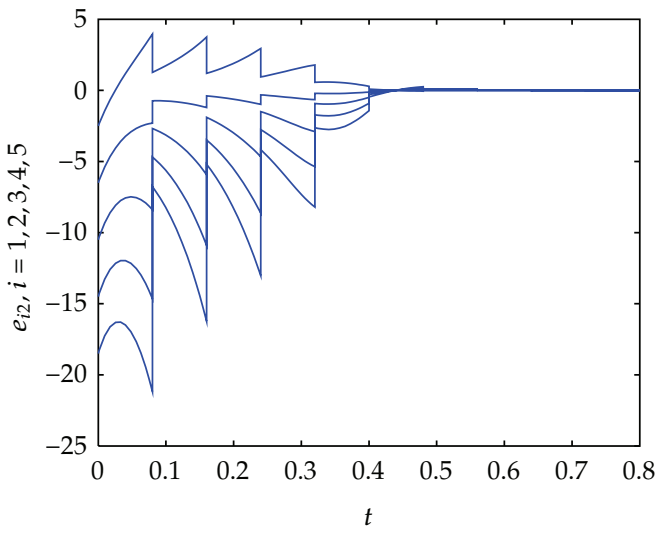

(b)

Figure 4: PS errors under the impulsive control.

The numerical results show that the adaptive-impulsive controlling scheme for the driveresponse complex network is effective in Theorem 3.1.

Remark 4.1. We only consider the impulsive control; the other conditions are chosen as above, and simulation results are shown in Figure 4. From Figures 2 and 4, it is easy to find that the control effect is not as well as the adaptive-impulsive control method.

Remark 4.2. In [31, 32], the authors investigated the PS of the drive-response dynamical networks model, but the time-varying coupling was not taken into account. Here, the PS of the drive-response time-varying coupling dynamical networks with time delay is studied by employing the adaptive-impulsive control. Furthermore, the proposed adaptive-impulsive control scheme is more effective than the adaptive control scheme in [31] and the impulsive control scheme in [32]. 


\section{Conclusion}

In this paper, the adaptive-impulsive PS of the drive-response time-varying coupling dynamical networks with time delay has been investigated. Based on the stability analysis of impulsive functional differential equations, some sufficient conditions for realizing the PS are established under the adaptive-impulsive control. And the results are a time delay independent stability criteria. Finally, numerical simulations have also been given to show effectiveness of the proposed method by an example of the unified chaotic systems. In the near future, we will extend the proposed method to discrete complex dynamical networks and discuss the delay-dependent case.

\section{Acknowledgments}

The author would like to thank the associate editor and the anonymous reviewers for their constructive comments and suggestions to improve the quality of the paper and Professor Qinsheng Bi for his help. This work was supported by the National Natural Science Foundation of China (nos. 10872080, 20976075, 10972091).

\section{References}

[1] D. J. Watts and S. H. Strogatz, "Collective dynamics of 'small-world' networks," Nature, vol. 393, no. 6684, pp. 440-442, 1998.

[2] A. -L. Barabási and R. Albert, "Emergence of scaling in random networks," Science, vol. 286, no. 5439, pp. 509-512, 1999.

[3] C. W. Wu, Synchronization in Complex Networks of Nonlinear Dynamical Systems, World Scientific, Singapore, 2007.

[4] B. Shen, Z. Wang, and Y. S. Hung, "Distributed $H_{\infty}$-consensus filtering in sensor networks with multiple missing measurements: the finite-horizon case," Automatica, vol. 46, no. 10, pp. 1682-1688, 2010.

[5] H. Dong, Z. Wang, D. W. C. Ho, and H. Gao, “Robust $H_{\infty}$ fuzzy output-feedback control with multiple probabilistic delays and multiple missing measurements," IEEE Transactions on Fuzzy Systems, vol. 18, no. 4, Article ID 5444946, pp. 712-725, 2010.

[6] H. Dong, Z. Wang, and H. Gao, “Observer-based $H_{\infty}$ control for systems with repeated scalar nonlinearities and multiple packet losses," International Journal of Robust and Nonlinear Control, vol. 20, no. 12, pp. 1363-1378, 2010.

[7] H. Dong, Z. Wang, D. W. C. Ho, and H. Gao, "Variance-constrained $H_{\infty}$ filtering for a class of nonlinear time-varying systems with multiple missing measurements: the finite-horizon case," IEEE Transactions on Signal Processing, vol. 58, no. 5, pp. 2534-2543, 2010.

[8] B. Shen, Z. Wang, Y. S. Hung, and G. Chesi, "Distributed $H_{\infty}$ filtering for polynomial nonlinear stochastic systems in sensor networks," IEEE Transactions on Industrial Electronics, vol. 58, no. 5, pp. 1971-1979, 2011.

[9] B. Shen, Z. Wang, and X. Liu, "Bounded $H_{\infty}$ synchronization and state estimation for discrete timevarying stochastic complex networks over a finite horizon," IEEE Transactions on Neural Networks, vol. 22, no. 1, pp. 145-157, 2011.

[10] P. De Lellis, M. di Bernardo, and F. Garofalo, "Synchronization of complex networks through local adaptive coupling," Chaos, vol. 18, no. 3, Article ID 037110, 8 pages, 2008.

[11] S. Wen, S. Chen, and W. Guo, "Adaptive global synchronization of a general complex dynamical network with non-delayed and delayed coupling," Physics Letters A, vol. 372, no. 42, pp. 6340-6346, 2008.

[12] S. Zheng, Q. Bi, and G. Cai, "Adaptive projective synchronization in complex networks with timevarying coupling delay," Physics Letters A, vol. 373, no. 17, pp. 1553-1559, 2009.

[13] X. F. Wang and G. Chen, "Pinning control of scale-free dynamical networks," Physica A, vol. 310, no. 3-4, pp. 521-531, 2002. 
[14] X. Li, X. Wang, and G. Chen, "Pinning a complex dynamical network to its equilibrium," IEEE Transactions on Circuits and Systems, vol. 51, no. 10, pp. 2074-2087, 2004.

[15] T. Chen, X. Liu, and W. Lu, "Pinning complex networks by a single controller," IEEE Transactions on Circuits and Systems, vol. 54, no. 6, pp. 1317-1326, 2007.

[16] W. Guo, F. Austin, S. Chen, and W. Sun, "Pinning synchronization of the complex networks with non-delayed and delayed coupling," Physics Letters A, vol. 373, no. 17, pp. 1565-1572, 2009.

[17] J. Zhou, L. Xiang, and Z. Liu, "Synchronization in complex delayed dynamical networks with impulsive effects," Physica A, vol. 384, no. 2, pp. 684-692, 2007.

[18] G. Zhang, Z. Liu, and Z. Ma, "Synchronization of complex dynamical networks via impulsive control," Chaos, vol. 17, no. 4, Article ID 043126, 9 pages, 2007.

[19] P. Li, J. Cao, and Z. Wang, "Robust impulsive synchronization of coupled delayed neural networks with uncertainties," Physica A, vol. 373, pp. 261-272, 2007.

[20] Q. Song, J. Cao, and F. Liu, "Synchronization of complex dynamical networks with nonidentical nodes," Physics Letters A, vol. 374, no. 4, pp. 544-551, 2010.

[21] H. Jiang and Q. Bi, "Impulsive synchronization of networked nonlinear dynamical systems," Physics Letters A, vol. 374, no. 27, pp. 2723-2729, 2010.

[22] Y. Tang, S. Y. S. Leung, W. K. Wong, and J. A. Fang, "Impulsive pinning synchronization of stochastic discrete-time networks," Neurocomputing, vol. 73, no. 10-12, pp. 2132-2139, 2010.

[23] M. Yang, Y. W. Wang, H. O. Wang, K. Tanaka, and Z. H. Guan, "Delay independent synchronization of complex network via hybrid control," in Proceedings of the American Control Conference (ACC '08), pp. 2266-2271, Seattle, Wash, USA, June 2008.

[24] K. Li and C. H. Lai, "Adaptive-impulsive synchronization of uncertain complex dynamical networks," Physics Letters A, vol. 372, no. 10, pp. 1601-1606, 2008.

[25] H. B. Jiang, "Hybrid adaptive and impulsive synchronisation of uncertain complex dynamical networks by the generalised Barbalat's lemma," IET Control Theory \& Applications, vol. 3, no. 10, pp. 1330-1340, 2009.

[26] Z.-H. Guan, D. J. Hill, and J. Yao, "A hybrid impulsive and switching control strategy for synchronization of nonlinear systems and application to Chua's chaotic circuit," International Journal of Bifurcation and Chaos, vol. 16, no. 1, pp. 229-238, 2006.

[27] R. Mainieri and J. Rehacek, "Projective synchronization in three-dimensional chaotic systems," Physical Review Letters, vol. 82, no. 15, pp. 3042-3045, 1999.

[28] L. X. Guo, Z. Y. Xu, and M. F. Hu, "Projective synchronization in drive-response networks via impulsive control," Chinese Physics Letters, vol. 25, no. 8, pp. 2816-2819, 2008.

[29] M. Hu, Y. Yang, Z. Xu, R. Zhang, and L. Guo, "Projective synchronization in drive-response dynamical networks," Physica A, vol. 381, no. 1-2, pp. 457-466, 2007.

[30] Y. Zhao and Y. Yang, "The impulsive control synchronization of the drive-response complex system," Physics Letters A, vol. 372, no. 48, pp. 7165-7171, 2008.

[31] M. Sun, C. Zeng, and L. Tian, "Projective synchronization in drive-response dynamical networks of partially linear systems with time-varying coupling delay," Physics Letters A, vol. 372, no. 46, pp. 6904-6908, 2008.

[32] X. Xu, Y. Gao, Y. Zhao, and Y. Yang, "The impulsive control of the projective synchronization in the drive-response dynamical networks with coupling delay," Advances in Neural Networks, vol. 6063, no. 1 , pp. 520-527, 2010.

[33] J. Lü and G. Chen, "A time-varying complex dynamical network model and its controlled synchronization criteria," IEEE Transactions on Automatic Control, vol. 50, no. 6, pp. 841-846, 2005.

[34] M. Chen, "Synchronization in time-varying networks: a matrix measure approach," Physical Review E, vol. 76, no. 1, Article ID 016104, 10 pages, 2007.

[35] P. Li and Z. Yi, "Synchronization analysis of delayed complex networks with time-varying couplings," Physica A, vol. 387, no. 14, pp. 3729-3737, 2008. 


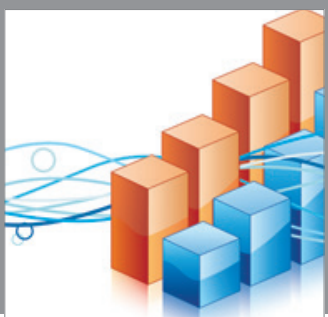

Advances in

Operations Research

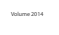

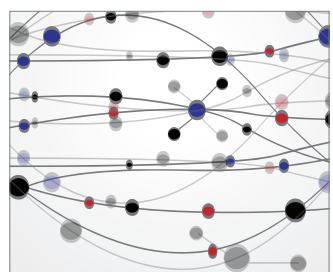

\section{The Scientific} World Journal
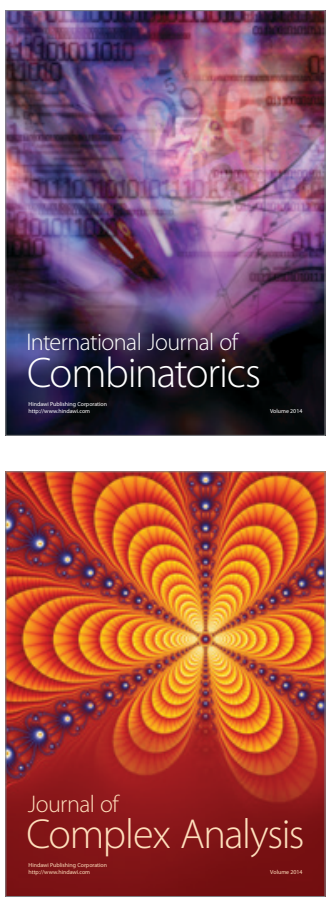

International Journal of

Mathematics and

Mathematical

Sciences
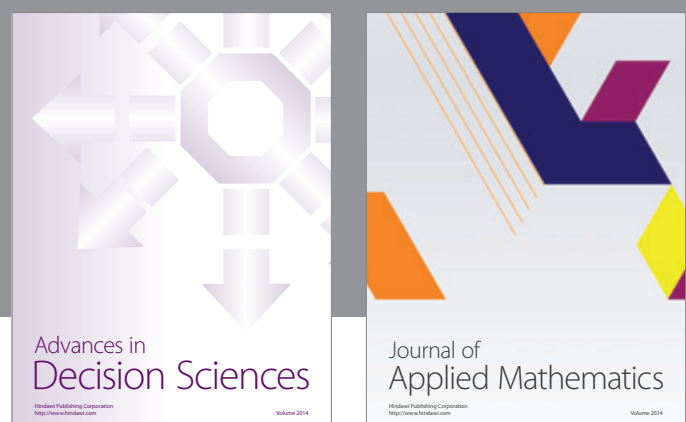

Journal of

Applied Mathematics
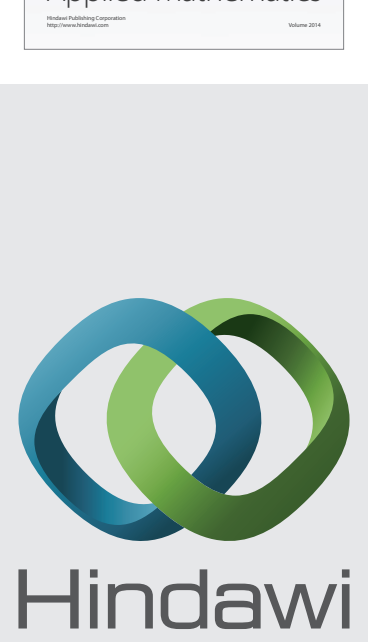

Submit your manuscripts at http://www.hindawi.com
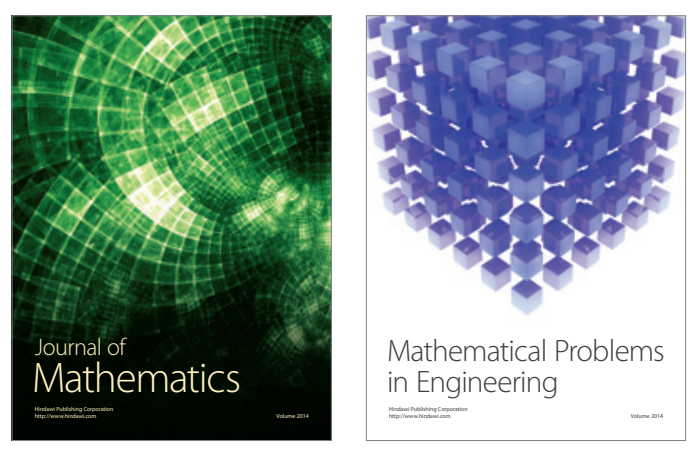

Mathematical Problems in Engineering
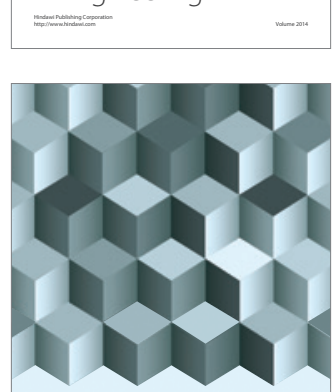

Journal of

Function Spaces
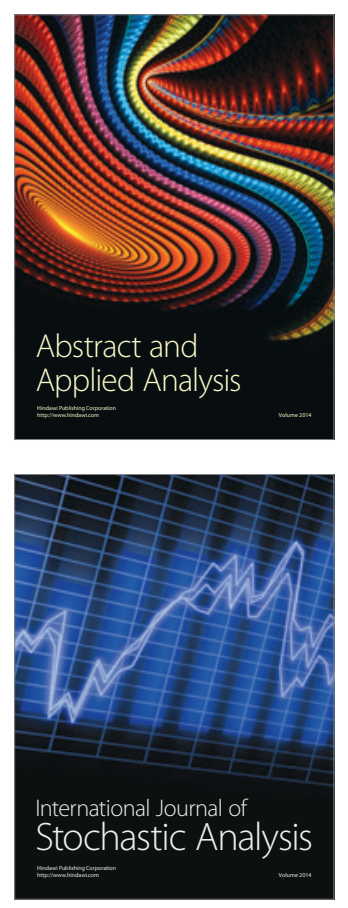

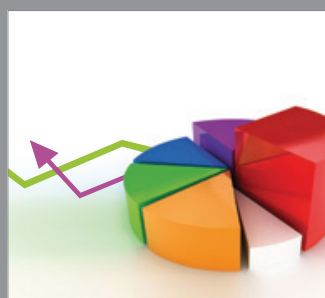

ournal of

Probability and Statistics

Promensencen
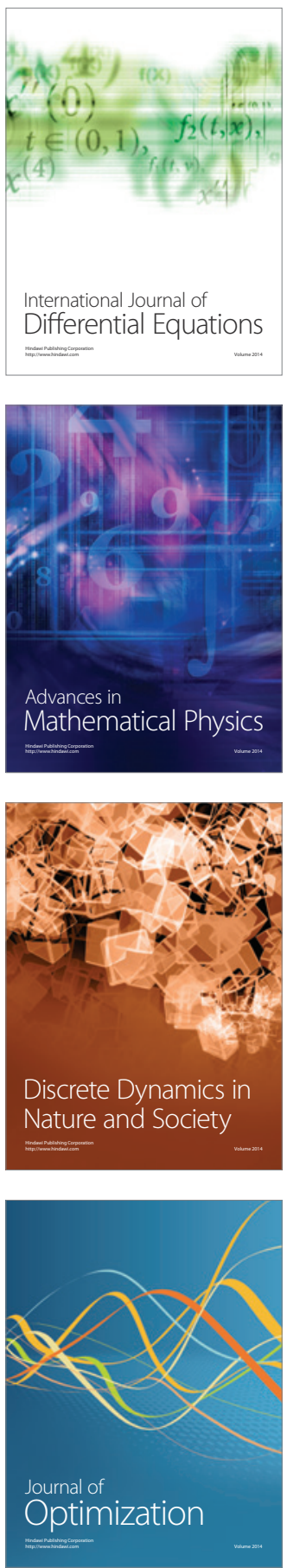